\title{
Synthesis and structural elucidation of new benzylidene imidazolidines and acridinylidene thiazolidines
}

T.G. SILVA. F.S.V. BARBOSA*. S.S.F. BRANDÃO*. M.C.A. LIMA*. S.L. GALDINO*. I.R. PITTA* and J. BARBE**

\author{
* Universidade Federal de Pernambuco. Departamento de Antibioticos 50.670-901 Recife. Brasil \\ ** GERCTOP - UMR CNRS 6009. Universite de la Mediterranee. Faculte de Pharmacie. 13385 Marseille \\ cedex 5. France.
}

\begin{abstract}
New benzylidene imidazolidine and acridinylidene thiazolidine derivatives were prepared from substituted imidazolidinones and substituted thio-imidazolidinones either by nucleophilic addition on cyanoacrylates or by condensation with arylaldehydes.
\end{abstract}

\section{Introduction}

It has been shown since a long time that acridines and azolidines are efficient drugs in infectious diseases. Thus we intended to prepare some corresponding condensed derivatives with the aim to obtain new drugs with synergistic activities [1-5].

\section{Results and discussion}

3-(4'-Bromo-benzyl)-4-thio-5-benzylidene-imidazolidin-2-ones, 4 and 5 , 1-methyl-3-(4'chloro-benzyl)-5-benzylidene-imidazolidin-2-ones, 8 and 9, and 1-methyl-2-thio-5-(4"bromo-benzylidene) imidazolidin-4-one, 11, were prepared by two different general routes. The first process used was condensation between 3-(4'-bromo-benzyl)-4-thio-imidazolidin-2one, $\mathbf{3}$, or 1-methyl-2-thio-imidazolidin-4-one, $\underline{10}$, with substituted benzaldehydes in acetic acid according to Johnson et al. [6] while the second one was the nucleophilic addition of 1 methyl-3-(4'-chloro-benzyl)-imidazolin-2,4-dione, 7, on selected aryl-substituted ethyl-(2cyano-3-phenyl)-acrylates [7] according to Daboun et al. [8].

Imidazolidin-2,4-dione, 1, 1-methyl-imidazolidin-2,4-dione, 6, and 1-methyl-2-thio-5-(4"bromo-benzylidene)-imidazolidin-4-one, 11 , were $\mathrm{N}(3)$-alkylated in the presence of potassium hydroxide which leads to the imidazolidine potassium salt capable to react with benzyl halides in hot alcoholic medium according to Finkbeiner [9]. 3-(4'-Bromo-benzyl)imidazolidin-2,4-dione, 2, 1-methyl-3-(4'-chloro-benzyl)-imidazolidin-2,4-dione, 7, and 1methyl-2-thio-3-benzyl-5-(4"-bromo-benzylidene)-imidazolidin-4-ones, $\underline{12}$ and $\underline{13}$, were obtained in this way.

3-(4'-Bromo-benzyl)-4-thio-imidazolidin-2-one, 3, was obtained from 3-(4'-bromo-benzyl)imidazolidin-2,4-dione, 2, using tetraphosphorous decasulfide according to Grishchuk et al. [10]. 
Synthetic pathways are portrayed in figure 1.<smiles>CN1CC(=O)N(Cc2ccc(Cl)cc2)C1=O</smiles><smiles>O=C1CNC(=O)N1</smiles><smiles>O=C1CNC(=O)N1Cc1ccc(Br)cc1</smiles>

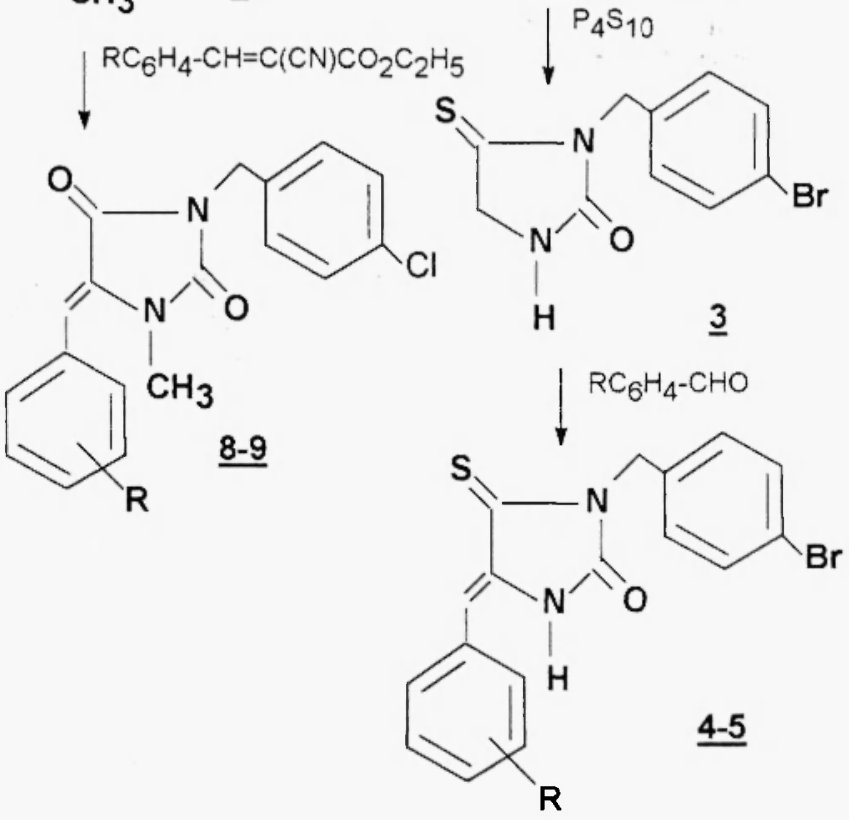<smiles>CN1CC(=O)NC1=S</smiles><smiles>CN1C(=O)C(=Cc2ccc(Br)cc2)N(C)C1=S</smiles>

Figure 1

The thiazolidine-dione derivatives 21-23, were only synthesized by nucleophilic addition of substituted thiazolidine-diones, $\underline{\mathbf{1}} \mathbf{\mathbf { 2 }} \mathbf{2 0}$, on 9-[ethyl-(2'-cyano)-acrylate]-acridine, 16 . Indeed, direct condensation of 9 -acridinaldehyde, $\underline{\mathbf{1 5}}$, with the substituted thiazolidine-diones $\underline{\mathbf{1 8}} \mathbf{- 2 0}$, did not led to the expected 3-benzyl-5-acridinylidene-thiazolidin-2,4-diones, 21-23, contrarily to that obtained with the imidazolidine reagents.

9-Methyl-acridine, 14 , was prepared from diphenylamine with zinc dichloride in acetic acid medium according to Tsuge et al. [11]. Subsequently, oxidation of 14 with pyridinium chlorochromate according to Mosher et al. [12] gave the 9-acridinaldehyde, 15 .

Synthetic pathways are portrayed in figure 2 .

Benzylidene imidazolidinones were isolated in a single isomer form. X-ray crystallographic studies and ${ }^{13} \mathrm{C}$ NMR have demonstrated the preferred $\mathrm{Z}$ configuration for 5 - 
arylidenethiazolidinones and 5-arylideneimidazolinones [13-15]. ${ }^{3} \mathrm{~J}_{\mathrm{CII}}$ coupling constant value between the ethylene proton and the carbon atom located at the $\alpha$ position of exocyclic bond is of special interest in this demonstration. Thus, in case of 1-methyl-3-(4'-chloro-benzyl)-5benzylidene-imidazolidin-2-one, $\mathbf{8}$, atter the methylene protons of the side chain being irradiated at $4.72 \mathrm{ppm}$, value of ${ }^{3} \mathrm{~J}_{\mathrm{Cl}}$ is $7.26 \mathrm{~Hz}$. This is in agreement with a $\mathrm{Z}$ configuration [16]. In contrast, 1-methyl-3-(4'-chloro-benzyl)-5-benzylidene-imidazolidin-2-one, 9, and 3benzyl-5-acridinylidene-thiazolidin-2,4-dione, 22, were isolated as isomeric mixtures. Isomers were readily identified by ${ }^{1} \mathrm{H}$ NMR. Actually, the ethylene proton is more deshielded in isomer $\mathrm{Z}$ than it is in isomer $\mathrm{E}$, owing to the cis-position of exocyclic carbonyl function. Finally, MS data fully agree with the structure proposed.<smiles>Cc1c2ccccc2nc2ccccc12</smiles><smiles>O=Cc1c2ccccc2nc2ccc(C(F)(F)F)cc12</smiles>

$\underline{14}$<smiles></smiles>

\section{$\underline{21}-\underline{23}$}

$\underline{15}$

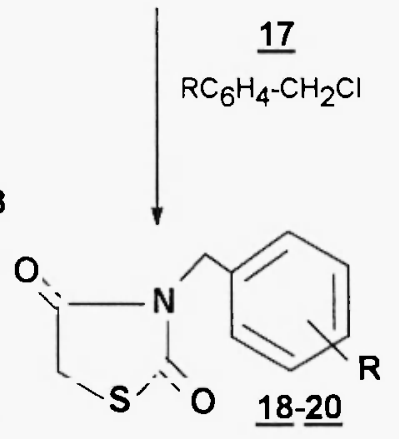

Figure 2 .

\section{Experimental}

Melting points were measured in capillary tube on Buchi (or Quimis) apparatus. Thin Layer Chromatography was performed on silicagel plates Merck $60 \mathrm{~F}_{254}$. Infrared spectra of $1 \% \mathrm{KBr}$ pellets were recorded on a Bruker IFS66 spectrometer (or Perkin Elmer 1310 spectrometer for $\underline{2}, 2, \underline{11}, \underline{12}$ and 13 ). ${ }^{1} \mathrm{H}$ NMR spectra were recorded on a Bruker AC $300 \mathrm{P}$ spectrophotometer (or Bruker AC 200 spectrophotometer for $2, \mathbf{8}, 9, \underline{\mathbf{1 2}}$ and $\underline{\mathbf{1 3}}$ ) in DMSO-d $\mathrm{D}_{6}$ or $\mathrm{CDCl}_{3}$ as solvent, with tetramethylsilane as internal standard. Mass spectra were recorded on DelsiNermag R 1010 C spectrometer (or HP 5897 for $\underline{\mathbf{2 1}}$ ).

The published chemical data on $\underline{2}$ [17], $\underline{6}, \underline{7}$ [3], $\underline{\mathbf{1 0}}$ [18], $\underline{19}$ [19] and $\underline{20}$ [2] have not been reported.

3-( $f^{\prime}$-Bromo-benzyl)-t-thio-imidazolidin-2-one, 3 .

3-(4'-Bromobenzyl)-imidazolidin-2,4-dione, $2,(6 \mathrm{~g}, 21 \mathrm{mmol})$ and tetraphosphorous decasulfide $(2.5 \mathrm{~g}, 11$ $\mathrm{mmol})$ are refluxed in anhydrous dioxane $(50 \mathrm{ml})$ for $40 \mathrm{~min}$ before zinc $(0.1 \mathrm{~g})$ and coal $(0.1 \mathrm{~g})$ be added. Heating is maintained $5 \mathrm{~min}$ more. The mixture is filtered and concentrated to the half. Crushed ice is added and the precipitate obtained is filtered. Crude is purified by column chromatography with benzene : methanol $(9 ; 1)$ as eluent. $\mathrm{C}_{1,0} \mathrm{H}_{9} \mathrm{~N}_{2} \mathrm{OS}$. Yield : $88 \%$. Mp : $188-9^{\circ} \mathrm{C}$. TLC, benzene : methanol $(9: 1), \mathrm{R}_{\mathrm{f}}: 0.57 . \mathrm{IR}^{-1}(\mathrm{KBr}): \mathrm{v}$ $3240,1755,1490,1450,1350,1230,880,780$. ${ }^{1} \mathrm{H}$ NMR $\left(\delta \mathrm{ppm}, \mathrm{CDCl}_{3}\right): 4.35\left(\mathrm{~d}, \mathrm{CH}_{2}-5 \mathrm{~J}=1 \mathrm{~Hz}\right) ; 4.99\left(\mathrm{~s}, \mathrm{CH}_{2}-\right.$ $\mathrm{Ph}$ ); 6.05 (s, NH); 7.36 (d, $2 \mathrm{H}$ benzyl, J = 8.6Hz); 7.45 (d. $2 \mathrm{H}$ benzyl, J = 8.5Hz). 
3-( $\downarrow^{\prime}$-lBromo-benzl'l)- $\downarrow$-thio-5-benzylidene-imidazolidin-2-ones, 4 , 5 : general procedure.

A mixture of benzaldehyde (1.2 mmol), 3-(4'-bromo-benzyl)-4-thio-inuidazolidin-2-one. 3 , (1.5 mmol) and melt sodium acetate $(0.45 \mathrm{~g})$ is heated at $120-140^{\circ} \mathrm{C}$ in acctic acid $(10 \mathrm{nll})$ for $5-6 \mathrm{~h}$. After cooling, precipitate is washed with water and recrystallized in acetic acid

3-(f'-Bromo-benzyl)-t-thio-5-(2"-chloro-benzylidene)-imidazolidin-t-one, 4

$\mathrm{C}_{17} \mathrm{H}_{12} \mathrm{BrClN} \mathrm{N}_{2} \mathrm{OS}$. Yield : $48 \%$. Mp : $218-9^{\circ} \mathrm{C}$. TLC. methylene chloride. $\mathrm{R}_{i}: 0.58$. IR cm $\mathrm{cm}^{-1}(\mathrm{KBr}): v 3224.1736$. 1639. 1487. 1305. 1 197. 764. ${ }^{1} \mathrm{H}$ NMR ( $\delta$ ppm. DMSO-d $): 5.04$ (s. $\left.\mathrm{CH}_{2}-\mathrm{Ph}\right): 7.19$ (s. CH): 7.31 (d. 2H benzyl. J $=8.7 \mathrm{~Hz}) ; 7.55$ (d. $2 \mathrm{H}$ benzyl. $\mathrm{J}=8.4 \mathrm{~Hz}): 7.41-7.45(\mathrm{~m} .2 \mathrm{H}$ benzylidene) $: 7.56-7.59$ ( $\mathrm{m}$. lH benzylidene): 7.747.77 (m. lH benzylidene): $11.5(\mathrm{NH})$. Ms. $\mathrm{m} / \mathrm{z}(\%): 371$ ( $\mathrm{M}^{+}-\mathrm{Cl}$ 71.4). 373(81.5), 169(100), 171(95.2). $90(14.7), 89(14.6)$

3-( $\downarrow^{\prime}$-Bromo-benzyl)- $\downarrow$-thio-5-( $/ "$-methoxy-benzylidene)-imidazolidin- - -one, 5

$\mathrm{C}_{18} \mathrm{H}_{15} \mathrm{BrN}_{2} \mathrm{O}_{2} \mathrm{~S}$. Yield : 48\%. Mp : 225-6 ${ }^{\circ} \mathrm{C}$. TLC. methylene chloride. $\mathrm{R}_{\mathrm{f}}: 0.50$. IR cm $\mathrm{cm}^{-1}(\mathrm{KBr}): 03237,1733$. 1646. 1597. 1347. 1259. 760. 'H NMR ( 8 ppm. DMSO-d $): 3.82\left(\mathrm{~s} . \mathrm{OCH}_{3}\right): 5.04\left(\mathrm{~s} . \mathrm{CH}_{2}-\mathrm{Ph}\right): 7,04$ (s. CH): 7.29 (d. $2 \mathrm{H}$ benzyl. $\mathrm{J}=8.1 \mathrm{~Hz}$ ); $7.55(\mathrm{~d} 2 \mathrm{H}$ benzyl. $\mathrm{J}=8.1 \mathrm{~Hz}) ; 7.02(\mathrm{~d} .2 \mathrm{H}$ benzylidenc. $\mathrm{J}=8.7 \mathrm{~Hz}$ ); $7.7(\mathrm{~d} .2 \mathrm{H}$ benzylidene. $\mathrm{J}=8.7 \mathrm{~Hz})$. Ms. $\mathrm{m} / \mathrm{z}(\%):$ 402( $\left.\mathrm{M}^{+} 100\right)$, 403(79.3). 404(93.8. 290(21.7), 233(15.7), 206(13), 169(38.5), 171(37.3). 89(6.5).

l-Methyl-3-( $4^{\prime}$-chloro-benzyl)-5-benzylidene-imidazolidin-2,4-ones, 8. 9 : general procedure.

A mixture of 1-methyl-3-(4'-chloro-benzyl)-imidazolidin-2.4-dione. 7, $(0.59 \mathrm{~g}, 2.5 \mathrm{mmol})$ and ethyl-(2-cyano-3phenyl)-acrylate $(2.7 \mathrm{mmol})$ is refluxed for $2-6 \mathrm{~h}$ in absolute ethanol $(20 \mathrm{ml})$ added with piperidine $(0.25 \mathrm{ml})$. After cooling, precipitates are purified by column chromatography and recrystallized in suitable solvents.

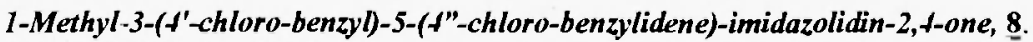

$\mathrm{C}_{18} \mathrm{H}_{14} \mathrm{Cl}_{2} \mathrm{~N}_{2} \mathrm{O}_{2}$ (purification by column chromatography with chloroform as eluent; recrystallisation in absolute ethanol). Yield : $18 \%$. Mp : $187-8^{\circ} \mathrm{C}$. TLC, chloroform : $n$-hexane $(1: 1), \mathrm{R}_{f}: 0.54 . \mathrm{IR} \mathrm{cm}^{-1}(\mathrm{KBr}): v 1754,1709$. 1629, 1403, 1086. 803. 'H NMR ( $\left.\delta \mathrm{ppm}, \mathrm{CDCl}_{3}\right) ; 3.21$ (s. $\left.\mathrm{NCH}_{3}\right) ; 4.71\left(\mathrm{~s} . \mathrm{CH}_{2}-\mathrm{Ph}\right) ; 6.18$ (s. $\left.\mathrm{CH}\right) ; 7.28($ d $2 \mathrm{H}$ benzyl, $\mathrm{J}=8.2 \mathrm{~Hz}$ ); 7.38 (d. $2 \mathrm{H}$ benzyl, $\mathrm{J}=8.3 \mathrm{~Hz}) ; 7.34(\mathrm{~d} .2 \mathrm{H}$ benzylidene. $\mathrm{J}=8.5 \mathrm{~Hz}) ; 7.92(\mathrm{~d} .2 \mathrm{H}$ benzylidenc. $\mathrm{J}=8.5 \mathrm{~Hz}) . \mathrm{Ms}, \mathrm{m} / \mathrm{z}(\%): 360\left(\mathrm{M}^{+} 58\right), 36 \mathrm{l}(11.3), 362(38.8), 166(17.1), 165(21), 150(34.1), 127(32.7), 125(100)$, $89(15.3)$.

l-Methyl-3-( + '-chloro-benzyl)-5-(2"-chloro-benzylidene)-imidazolidin-2,4-one, 9.

$\mathrm{C}_{18} \mathrm{H}_{14} \mathrm{Cl}_{2} \mathrm{~N}_{2} \mathrm{O}_{2}$ (purification by column chromatography with chloroform / n-hexane ( $8: 2$ ) as eluent: recrystallization in absolute ethanol). Yield : $15 \%$. Mp : $164-5^{\circ} \mathrm{C}$. TLC, chloroform : $n$-hexane (1:1), $\mathrm{R}_{f}: 0.47$. IR cm ${ }^{-1}(\mathrm{KBr}): \cup 1765.1715,1650,1440.1405,1100,850 .{ }^{1} \mathrm{H}$ NMR ( $\delta \mathrm{ppm}$. DMSO-d 6 ) Z/E 56/44\% : 3.16/2.77 (s, $\left.\mathrm{NCH}_{3}\right) ; 4.68 / 4.60\left(\mathrm{~s}, \mathrm{CH}_{2}-\mathrm{Ph}\right) ; 6.67 / 6.50(\mathrm{~s}, \mathrm{CH}) ; 7.32-7.44$ (m, arom.) 7.81-7.86 (m, benzylidene). Ms, $\mathrm{m} / \mathrm{z}(\%)$ : 360(M 1.8), 361(1), 362(1.1), 325(54), 150(16.9), 127(47.4), 125(100), 99(9.2), 89(27.1). ${ }^{13} \mathrm{C}$ NMR $(\delta$ ppm. DMSO-d $\left.\mathrm{d}_{5}, \mathrm{DEPT}\right) \mathrm{Z} / \mathrm{E}: 26.4 / 26.6(\mathrm{NCH}), 40.9 / 41.3(\mathrm{CH} 2), 107.1 / 111.1$ (CH ethylenique), 126.3/126.7 (CH), 128.4/128.5 (2CH), 128.8/129.1 (CH), 129.5/129.6 (2CH), 129.9/130.1 (CH), 130.6/130.9 (C), 131/131.1 (C), 131.7/131.9(CH), 132.1/132.2(C), 132.8/133.2(C), 135.1/135.2(C), 152.9/154.7 (CO), 160.8/162.6 (CO). Ms, m/z (\%) : 360(M 1.8), 36l(1) 362(1.1), 325(54), 327(16.4), 150(16.9), 125(100), 127(47.4), 89(27.1).

\section{l-Methyl-2-thio-5-( $f^{\prime \prime}$-bromo-benzylidene)-imidazolidin-4-one, 11.}

A mixture of 1-methyl-2-thio-imidazolidin-4-one, $10,(1.3 \mathrm{~g} .0 .01 \mathrm{mmol}), 4$-bromo-benzaldehyde $(2.78 \mathrm{~g}, 0.015$ mmol) and melt sodium acetate $(2.5 \mathrm{~g})$ is heated at $1+0^{\circ} \mathrm{C}$ in glacial acetic acid for $3 \mathrm{~h}$. After cooling, the precipitate obtained is washed with water then with chloroform.

$\mathrm{C}_{11} \mathrm{H}_{9} \mathrm{BrN}_{2} \mathrm{OS}$. Yield : $60 \%$. Mp : $212^{\circ} \mathrm{C}$. TLC, chloroform : methanol $(94: 6), \mathrm{R}_{\mathrm{f}} 0.83$. IR cm $\mathrm{cm}^{-1}(\mathrm{KBr}): \mathrm{v} 3130$, $1735,1620,1580,1485,1370,1160,1105,770 .{ }^{1} \mathrm{H}$ NMR $\left(\delta \mathrm{ppm}, \mathrm{DMSO}-\mathrm{d}_{6}\right): 3.44\left(\mathrm{~s}, \mathrm{NCH}_{3}\right) ; 6.66(\mathrm{~s}, \mathrm{CH}) ; 7.56$ (d, $2 \mathrm{H}$ benzylidene, $\mathrm{J}=8.2 \mathrm{~Hz}) ; 7.96(\mathrm{~d}, 2 \mathrm{H}$ benzylidene, $\mathrm{J}=8.2 \mathrm{~Hz}) ; 13.42(\mathrm{~s}, \mathrm{NH}){ }^{13} \mathrm{C}$ NMR $(\delta \mathrm{ppm}$, DMSO$\left.\mathrm{d}_{6}, \mathrm{DEPT}\right): 29.7(\mathrm{NCH} 3), 118.4,122.6,130.4,131,131.7,132.5,162.6(\mathrm{CO}), 176.1(\mathrm{CS})$.

I-Methyl-2-thio-3-benzyl-5-(4"-bromo-benzylidene)-imidazolidin-4-ones, 12,13 : general procedure.

1-Methyl-2-thio-5-(4"-bromo-benzylidene)-imidazolidin-4-one, $11,(0.59 \mathrm{~g}, 2 \mathrm{mmol})$ is dissolved in methanol $(2$ $\mathrm{ml}$ ) before potassium hydroxide $(0.12 \mathrm{~g})$ dissolved in methanol $(3 \mathrm{ml})$ be added with stirring. A few time (10 $\mathrm{min})$ later, benzyl halide $(2.5 \mathrm{mmol})$ is added dropwise. The mixture is left at room temperature for $24 \mathrm{~h}$. The precipitate obtained is filtered, before to be washed with water then with ethyl ether. Compounds isolated are of acceptable purity and were analysed without further recrystallization. 
1-Methyl-2-thio-3-benzil-5-(4"-bromo-benzlidene)-imidazolidin-t-one, 12.

$\mathrm{C}_{18} \mathrm{H}_{15} \mathrm{BrN} \mathrm{N}_{2} \mathrm{OS}$. Yield : $80 \%$. Mp : 209-211 ${ }^{\circ} \mathrm{C}$. TLC. chloroform : methanol $(96: 4), \mathrm{R}_{i}: 0.9 . \mathrm{IR} \mathrm{cm}^{-1}(\mathrm{KBr}): 0$ $1680,1620,1590.1455 .1435 .1390 .1170,1020.720 .{ }^{1} \mathrm{H}$ NMR $\left(\delta \mathrm{ppm} . \mathrm{DMSO}-\mathrm{d}_{\mathrm{f}}\right): 3.29\left(\mathrm{~s} . \mathrm{NCH}_{3}\right): 4.57(\mathrm{~s}$. $\mathrm{CH}_{2}$ ); 6.79 (s, CH): $7.31-7.48$ (m. $5 \mathrm{H}$ benzyl): 7.61 (d. $2 \mathrm{H}$ benzylidene. J = 8.4Hz); 8.17 (d. $2 \mathrm{H}$ benzylidene. $\mathrm{J}=$ 8.4Hz). Ms. m/z (\%) : 386( $\mathrm{M}^{+}$18). 388(20). 353(5.2). 274(8.7). 226(7.6), 209(17.9). 194(46.2). 196(51.5). 115(18.7), $91(100), 65(33.8)$.

1-Methyl-2-thio-3-(4'-bromo-benzy')-5-(4"-bromo-benzylidene)-imidazolidin-4-one, 13.

$\mathrm{C}_{18} \mathrm{H}_{1 .} \mathrm{Br}_{2} \mathrm{~N}_{2} \mathrm{OS}$. Yield : $85 \%$. Mp : $223-224^{\circ} \mathrm{C}$. TLC. chloroform : methanol $(96: 4) . \mathrm{R}_{\mathrm{f}} ; 0.9$. IR cm ${ }^{-1}(\mathrm{KBr}): \mathrm{v}$ 1690, 1610, 1580, 1485, 1450, 1435, 1320, 1160. 1010, 890. ' ${ }^{1} \mathrm{H}$ NMR $\left(\delta \mathrm{ppm} . \mathrm{DMSO}-\mathrm{d}_{6}\right): 3.29\left(\mathrm{~s} . \mathrm{NCH}_{3}\right): 4.55$ (s. $\left.\mathrm{CH}_{2}\right) ; 6.79(\mathrm{~s} . \mathrm{CH}) ; 7.43$ (d. $2 \mathrm{H}$ benzyl, J $=8.5 \mathrm{~Hz}$ ): 7.54 (d. $2 \mathrm{H}$ benzyl. J $=8.5 \mathrm{~Hz}$ ): 7.61 (d. $2 \mathrm{H}$ benzylidene. $\mathrm{J}$ $=8.6 \mathrm{~Hz}) ; 8.17(\mathrm{~d} .2 \mathrm{H}$ benzylidene. $\mathrm{J}=8.6 \mathrm{~Hz})$. Ms. $\mathrm{m} / \mathrm{z}(\%): 46+\left(\mathrm{M}^{+} 0.51\right), 466(0.7), 468(0.65), 352(7.3)$. 295(6.6), 226(12.9), 209(22.4). 194(39.7). 169(49), 115(26.7), 90(60.6), 89(93.7), 63(100).

9-Methyl-acridine, 14 .

A mixture of diphenvlamine $(2.5 \mathrm{~g} .14 .7 \mathrm{mmol})$, zinc dichloride $(10 \mathrm{~g})$ and acetic acid $(2.5 \mathrm{ml})$ is heated at $220^{\circ} \mathrm{C}$ for $6 \mathrm{~h}$. The mixture is acidified with $10 \%$ sulfuric acid before to be made alkaline with $30 \%$ ammonia. The crude is extracted with toluene and purified by flash chromatography on silica with $n$-hexane : ethyl acetate (7:3) as eluent. $\mathrm{C}_{14} \mathrm{H}_{11} \mathrm{~N}$. Yield : $40 \% . \mathrm{Mp}: 115-7^{\circ} \mathrm{C} / 118^{\circ} \mathrm{C}[13]$. TLC, $n$-hexan : ethyl acetate (7:3), $\mathrm{R}_{\mathrm{f}}: 0.59$. ${ }^{1} \mathrm{H}$ NMR $\left(\delta \mathrm{ppm}, \mathrm{DMSO}-\mathrm{d}_{6}\right) ; 3.13\left(\mathrm{~s} . \mathrm{CH}_{3}\right) ; 7.60-7.66\left(\mathrm{~m} .2 \mathrm{H}_{\text {arom }}\right) ; 7.81-7.87\left(\mathrm{~m} .2 \mathrm{H}_{\text {arom }}\right) ; 8.12-8.17\left(\mathrm{~m}_{2} 2 \mathrm{H}_{\text {arom }}\right)$; 8.38-8.42(m. $\left.2 \mathrm{H}_{\text {arom }}\right)$.

\section{9-Acridinaldehyde, 15.}

A mixture of 9-methyl-acridine, $\underline{1},(1.7 \mathrm{~g} .8 .9 \mathrm{mmol})$, magnesium sulfate $(5.1 \mathrm{~g})$, pyridinium chlorochromate (PCC) $(2.04 \mathrm{~g}, 9.4 \mathrm{mmol})$ and anhydrous methylene chloride $(5 \mathrm{l} \mathrm{ml})$ is stirred under nitrogen pressure at room temperature for $18 \mathrm{~h}$. The crude is extracted with ethyl ether. After solvent evaporation. compound is purified by flash chromatography on silica with n-hexane : ethyl acetate $(6: 4)$ as eluent. $\mathrm{C}_{14} \mathrm{H}_{9} \mathrm{NO}$. Yield : $55 \%$. Mp : $151-$ $152^{\circ} \mathrm{C} / 1+7^{\circ} \mathrm{C}[14]$. TLC, $n$-hexan : ethyl acetate $(6: 4), \mathrm{R}_{\mathrm{f}}: 0.35 .{ }^{1} \mathrm{H}$ NMR $\left(\delta \mathrm{ppm}\right.$. DMSO-d $\left.\mathrm{d}_{6}\right) ; 11.67$ (s. CHO); 7.22-7.28 (m. $\left.2 \mathrm{H}_{\text {arom }}\right) ; 7.52-7.55$ (m. $\left.2 \mathrm{H}_{\text {arom }}\right) ; 7.70-7.75\left(\mathrm{~m} .2 \mathrm{H}_{\text {arom }}\right) ; 8.22-8.25\left(\mathrm{~m}, 2 \mathrm{H}_{\text {arom }}\right)$.

\section{9-[Ethyl-(2'-cyano)-acrylate/-acridine, 16.}

A mixture of 9-acridinaldehyde, $15,(2.2 \mathrm{~g}, 10.5 \mathrm{mmol})$, ethyl cyanoacetate $(3 \mathrm{ml})$, piperidine $(250 \mu \mathrm{l})$ and anhydrous benzene $(50 \mathrm{ml})$ is refluxed at $110^{\circ} \mathrm{C}$ for $8 \mathrm{~h}$ After cooling, the precipitate obtained is purified by flash chromatography on silica with $n$-hexane : ethyl acetate $(6: 4)$ as eluent. $\mathrm{C}_{19} \mathrm{H}_{14} \mathrm{~N}_{2} \mathrm{O}_{2}$. Yield : $49 \%$. $\mathrm{Mp}$ : $170-171^{\circ} \mathrm{C}$. TLC, $n$-hexane ethyl acetate $(7: 3), \mathrm{R}_{\mathrm{f}}: 0.44 \mathrm{IR} \mathrm{cm}^{-1}(\mathrm{KBr}): \cup 3431,2200,1727,1619,1270.763$. ${ }^{1} \mathrm{H}$ NMR ( $\delta$ ppm, DMSO-d 6 ): $1.40\left(\mathrm{t}, \mathrm{CH}_{3}, \mathrm{~J}=7.2 \mathrm{~Hz}\right) ; 4.45\left(\mathrm{q}, \mathrm{CH}_{2}, \mathrm{~J}=7.2 \mathrm{~Hz}\right) ; 7.69-7.75(\mathrm{~m}, 2 \mathrm{H}) ; 7.91-7.97$ (m. $2 \mathrm{H}) ; 8.11$ (d, $2 \mathrm{H}, \mathrm{J}=8.7 \mathrm{~Hz}) ; 8.26$ (d. $2 \mathrm{H}, \mathrm{J}=8.4 \mathrm{~Hz}) ; 9.35(\mathrm{~s}, \mathrm{CH}) . \mathrm{Ms}, \mathrm{m} / \mathrm{z}(\%): 302\left(\mathrm{M}^{+} 25.1\right), 303(5.4)$, 229(100), 203(19.7), 175(5.3), 101(4.5), 75(5.9), 63(5.1).

3-(4'-Methyl-benzyl)-thiazolidin-2,4-dione, 18.

A solution of sodium hydroxide $(1.9 \mathrm{~g})$ in $10 \mathrm{ml}$ of ethanol : water mixture $(6: 4)$ is added dropwise under stirring to a suspension of thiazolidine-2,4-dione, 17 , in $10 \mathrm{ml}$ of the same ethanol : water mixture. A few time later $(10 \mathrm{~min})$, the substituted benzyl chloride $(7 \mathrm{ml})$ is adcied and the mixture is stirred for $5 \mathrm{~min}$ before to be refluxed for $24 \mathrm{~h}$. After cooling at room temperature, the expected compound is precipitated by addition of crushed ice before to be purified by flash chromatography on silica with chloroform : methanol $(92: 8)$ as eluent. $\mathrm{C}_{11} \mathrm{H}_{11} \mathrm{NO}_{2} \mathrm{~S}$. Yield : $21 \%$. Mp : $70-72^{\circ} \mathrm{C}$. TLC chloroform : methanol $(92: 8) \mathrm{R}_{\mathrm{f}}: 0.68$. IR $\mathrm{cm}^{-1}(\mathrm{KBr}): \mathrm{v} 2996$, $1756,1730,1676,1514,1+28,1378,1337,1058,751$. 'H NMR ( $\delta$ ppm, DMSO-d 6$): 2.27\left(\mathrm{~s}, \mathrm{CH}_{3}\right) ; 4.26(\mathrm{~s}$, $\left.\mathrm{CH}_{2}\right) ; .62\left(\mathrm{~s}, \mathrm{CH}_{2}\right.$ phenacyl), $7.14(\mathrm{~s}, 4 \mathrm{H}$, arom. $)$.

3-Benzyl-5-acridinylidene-thiazolidin-2,4-diones, $21-23$; general procedure.

Benzylthiazolidine, $18-20(0.9 \mathrm{mmol})$ and 9 -[ethyl-(2'-cyano)-acrylate]-acridine. $16,(0.9 \mathrm{mmol})$ are dissolved in absolute ethanol $(8 \mathrm{ml})$. The solution is refluxed for $4 \mathrm{~h}$ in the presence of small amount of piperidine as catalyst. The precipitate obtained is filtered and washed with water. Compounds isolated are of acceptable purity and were analysed without further recrystallization. 
3-(t'-Methyl-benzll)-5-racridinylidene-thiazolidin-2, -dione, 21.

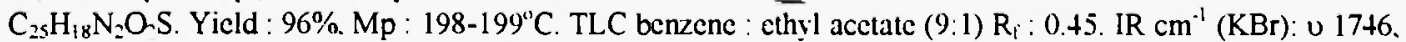
1697, 1630. 1378. 1339. 1149.758. ${ }^{1} \mathrm{H}$ NMR (o ppm. $\left.\mathrm{CDCl}_{3}\right): 2.37\left(\mathrm{~s} . \mathrm{CH}_{3}\right) .+92\left(\mathrm{~s} . \mathrm{CH}_{2}\right): 7.2 \mathrm{l}$ (d. $2 \mathrm{H}$ benzyl. J $=7.8 \mathrm{~Hz}$ ) $.7 .42(\mathrm{~d} .2 \mathrm{H}$ benzyl. $\mathrm{J}=8.1 \mathrm{~Hz}$ ). $7.57-7.62(\mathrm{~m} .2 \mathrm{H}$ acridin). $7.8-7.85(\mathrm{~m} .2 \mathrm{H}$ acridin). $7.96(\mathrm{~d} 2 \mathrm{H}$ acridin. $\mathrm{J}=7.8 \mathrm{~Hz}$ ). 8.29 (d. $2 \mathrm{H}$ acridin. $\mathrm{J}=8.7 \mathrm{~Hz}) .8 .69\left(\mathrm{~s} .1 \mathrm{H} . \mathrm{CH}\right.$ ). Ms. $\mathrm{m} / \mathrm{z}(\%): 410\left(\mathrm{M}^{+} 98.1\right) .411(29.3)$. 305(24.2). 235(52.9). 190(9). 105(100). 77(8.3).

3-(t'-Bromo-benz:ll)-5-acridinylidene-thiazolidin-2.t-done. 22.

$\mathrm{C}_{2,1} \mathrm{H}_{15} \mathrm{BrN} \mathrm{N}_{2} \mathrm{O}_{2} \mathrm{~S}$. Yield : $48 \%$. Mp : 258-260" C. TLC ethyl acctate : $n$-hexanc (7:3). $\mathrm{R}_{\mathrm{f}}: 0.53 . \mathrm{IR} \mathrm{cm}^{-1}(\mathrm{KBr}): \mathrm{v}$ 3+19. 1745. 1694. 1625. 1378. 1334. 11+9. 760. 'H NMR (s ppm. DMSO-d $)$ Z/E 78/22\% : 4.86/4.57 (s. CH.): 7.39/7.10 (d. $2 \mathrm{H}$ benzyl. J = 8.7/8.4Hz): 7.60/7.46 (d. $2 \mathrm{H}$ benzyl. J $=8.7 \mathrm{~Hz}$ ): 7.66-7.72 (Z, m. $2 \mathrm{H}$ acridin), 7.897.95 (Z. m. $2 \mathrm{H}$ acridin). 8.14 (Z. d. $2 \mathrm{H}$ acridin. $\mathrm{J}=8.1 \mathrm{~Hz}$ ). 8.24 (Z. d. $2 \mathrm{H}$ acridin. $\mathrm{J}=8.4 \mathrm{~Hz}$ ), $8.78 / 8.37$ (s. CH).

3-(4'-Nitro-benavl)-5-acridinylidene-thiazolidin-2, t-dione, 23.

$\mathrm{C}_{34} \mathrm{H}_{15} \mathrm{~N}_{3} \mathrm{O} . \mathrm{S}$. Yicld : $60 \%$. Mp : $238-240^{\circ} \mathrm{C}$. TLC $n$-hexane : ethyl acetate $(6: 4) \mathrm{R}_{\mathrm{f}}: 0.57$. IR $\mathrm{cm}^{-1}(\mathrm{KBr}): \mathrm{v}$ 3422, 1747. 1694. 1629, 1606, 1530, 1379, 1340, 11+9. 760. ' $\mathrm{H}$ NMR ( $\delta \mathrm{ppm}$. DMSO-d $\left.\mathrm{d}_{6}\right) 5.03\left(\mathrm{~s} . \mathrm{CH}_{2}\right): 7.72(\mathrm{~d}$ $2 \mathrm{H}$ benzyl. $\mathrm{J}=8.7 \mathrm{~Hz}$ ): 8.27 (d. $2 \mathrm{H}$ benzyl. $\mathrm{J}=8.4 \mathrm{~Hz}$ ): 7.68-7.73 (m. $2 \mathrm{H}$ acridin), 7.90-7.96 (m. $2 \mathrm{H}$ acridin), $8.17(\mathrm{~d} 2 \mathrm{H}$ acridin. $\mathrm{J}=8.7 \mathrm{~Hz}), 8.25(\mathrm{~d} .2 \mathrm{H}$ acndin. $\mathrm{J}=8.7 \mathrm{~Hz}) .8 .81(\mathrm{~s}, 1 \mathrm{H} . \mathrm{CH})$.

\section{Acknowledgments}

The CAPES/COFECUB (Coordenação de Aperfeiçoamento de Pessoal do Ensino Superior/COmite Fiançais d'Evaluation et de Cooperation Universitaire avec le Brésil) are gratefully acknowledged for their support to the join research programme.

\section{References}

[1] H.M. SALAMA. I.M. LABOUTA and M. MAA. Alexandna J. Pharm. Sc.. 4. 4t-46 (1990).

[2] S.S.F. BRANDÃO. J. A. ROCHA FILHO. J. CHANTEGREL. J.F.C. ALBUQUERQUE. E.A. XIMENES. S.L. GALDINO. I.R. PITTA, M. PERRISSIN and C. LUU-DUC, Ann. Pharm. Fr., 55, 206-211 (1997).

[3] J.F.C. ALBUQUERQUE, A.J. ROCHA FILHO, S.S.F. BRANDÃO. M.C.A LIMA. E.A. XIMENES. S.L. GALDINO, I.R. PITTA. J. CHANTEGREL, M. PERRISSIN and C. LUU-DUC. II Farmaco, 54. 77-82 (1999).

[4] A. ALBERT and R. GOLDACRE, Naturc, 162,95 (1948)

[5] A. ALBERT. S.D. RUBBO, R.J. GOLDACRE. M.E. DAVEY and J.D. STONE. Brit. J. exptl Pathol.. 26. $160(19+5)$

[6] T.B. JOHNSON and W.M. SCOTT, J. amer. chem. Soc., 37, 1846-1856 (1915)

[7] W.M. PHILLIPS and D.J. CURRIE, Can. J. Chem., 47, 3137-31+6 (1969).

[8] H.A.F. DABOUN, S.E. ABDOU, M.M. HUSSEIN and M.H. ENALGDI, Synthesis, 502-504 (1982).

[9] H. FINKBEINER, J. Org. Chem., 30. 341+-3+19 (1965).

[10] A.P. GRISHCHUK, S.N. BARANOV, T.E. GORIZDRA and I.D. KOMARITSA. Zh. Prikl. Khim., $\mathbf{4 0 .}$ 1389-1390 (1967) in Chem. Abstr. 67, $116869^{\circ}$ (1967).

[11] O TSUGE, M. NISHINOHARA and M. TASHIRO. Bull. Chem. Soc. Japan, 36, 1477-1485 (1963).

[12] M.D. MOSHER and N.R. NATALE, J. Heterocycl. Chem.. 32. 779-781 (1995).

[13] S.F. TAN, K.P. ANG and Y.F. FONG, J. Chem. Soc., Perkin Trans. II. 1941-194t (1986).

$[1+]$ J.F.C. ALBUQUERQUE, S.L. GALDINO, J. CHANTEGREL, F. THOMASSON. I.R. PITTA and C. LUU-DUC, Ann. Pharm. Fr.. in press.

[15] C.A. DE SIMONE, J. ZUKERMAN-SCHPECTOR, M.A. PEREIRA, C. LUU-DUC, I.R. PITTA, S.L. GALDINO and E.L.C. AMORIM. Acta Cryst., C51, 2620-2621 (1995).

[16] G.J. KARABATSOS and C.E. ORZECH, J. amer. chem. Soc, 87, 560-562 (1965).

[17] E.L. AMORIM, S.S.F. BRANDÃO, C.O.M. CAVALCANTI, S.L.GALDINO, I.R. PITTA and C. LUUDUC. Ann. Pham. Fr., 50,103-111 (1992).

[18] S.S.F. BRANDÃO, V.L.M. GUARDA, I.R. PITTA, J. CHANTEGREL, M. PERRISSIN, V.M. SOUZA, S.L. GALDINO, F. THOMASSON, M.C.A.LIMA, L.F.C.C. LEITE and C. LUU-DUC. Boll. Chim. Farm., 139, 54-58 (2000).

[19] D.L.B. COSTA, J. CHANTEGREL, M.C.A. LIMA, J.F.C. ALBUQUERQUE, R.M.O.C. LIMA, S.L. GALDINO, I.R. PITTA and C. LUU-DUC. J. Pharm. Belg., 50, 5-10 (1995).

\section{Received on October 10, 2001}

